\title{
Toxicidad de extractos de Carya illinoinensis (Fagales: Junglandaceae) contra Meloidogyne incognita (Tylenchida: Heteroderidae) en tomate
}

\section{Toxicity of Carya illinoinensis (Fagales: Junglandaceae) extracts against Meloidogyne incognita (Tylenchida: Heteroderidae) in tomato}

\author{
Melchor Cepeda-Siller ${ }^{1}$, José Miguel García-Calvario ${ }^{1}$, Agustín Hernández-Juárez ${ }^{1 *}$, Yisa María \\ Ochoa-Fuentes $^{1}$, Fabiola Garrido-Cruz ${ }^{1}$, Ernesto Cerna-Chávez ${ }^{1}$, Miriam Desireé Dávila-Medina ${ }^{2}$ \\ ${ }^{1}$ Departamento de Parasitología, Universidad Autónoma Agraria Antonio Narro, Calzada Antonio Narro 1923. CP. 25315. \\ Buenavista, Saltillo, Coahuila. México. \\ ${ }^{2}$ Profesor invitado. \\ *Autor de correspondencia: chinoahj14@hotmail.com
}

Nota científica recibida: 28 de junio de 2016 aceptada: 23 de marzo de 2017

RESUMEN. Meloidogyne incognita es importante en el cultivo de tomate, por su capacidad para reducir el rendimiento y la calidad del fruto. El objetivo fue evaluar la actividad tóxica de extractos de cáscara y ruezno de nogal pecanero (Carya illinoinensis), sobre el nematodo agallador ( $M$. incognita) en plantas de tomate en invernadero. Se evaluaron los extractos vegetales de ruezno y cáscara de nogal pecanero en siete tratamientos con cuatro repeticiones bajo un diseño en bloques completos al azar, mediante la valoración de la densidad de población de juveniles de segunda generación, y se determinó la eficiencia de los tratamientos. Se encontró efecto de la aplicación de los extractos de nogal sobre $M$. incognita; sobresaliendo los extractos de ruezno acuoso con 99 y $97 \%$ de reducción de la población en concentraciones 1:50 y 1:75; seguido del extracto de ruezno etanólico con $87 \%$ en la concentración 1:50 y $71 \%$ en la concentración $1: 75$.

Palabras clave: Control, extractos vegetales, nematodo agallador, nogal pecanero, Solanum lycopersicum

ABSTRACT. Meloidogyne incognita is important in tomato cultivation because of its ability to reduce fruit yield and quality. The objective was to evaluate the toxic activity of extracts from the pecan (Carya illinoinensis) nut shell and husk on the southern root-knot nematode ( $M$. incognita) in greenhouse-grown tomato plants. Plant extracts of pecan nut husk and shell were evaluated in seven treatments with four replications under a randomized complete block design, by assessing the population density of second-generation juveniles, and the efficiency of the treatments was determined. Applying pecan nut extracts on $M$. incognita was found to have an effect, highlighted by the aqueous shell extracts with a 99 and $97 \% M$. incognita population reduction at concentrations of $1: 50$ and $1: 75$, followed by the ethanol husk extract with $87 \%$ at the $1: 50$ concentration and $71 \%$ at the $1: 75$ concentration.

Key words: Control, plant extracts, southern root-knot nematode, pecan nut, Solanum lycopersicum

\section{INTRODUCCIÓN}

El cultivo del tomate (Solanum lycopersicum L.), es una de las hortalizas más importantes en el mundo. Ocupa el segundo lugar en importancia en México en participación del valor de la producción nacional de hortalizas, con una producción de 2875 $164.08 \mathrm{t}$, destacando como principal productor el es- tado de Sinaloa con 867832.04 t (SAGARPA-SIAP 2016).

Meloidogyne incognita (Kofoid y White) Chitwood es uno de los patógenos más importantes en el tomate, el cual afecta la producción mundial, ocasionando pérdidas entre 28 y $68 \%$ al año (Nicol et al. 2011, Jones et al. 2013). El cual es un endoparásito sedentario de penetración total, que presenta una 


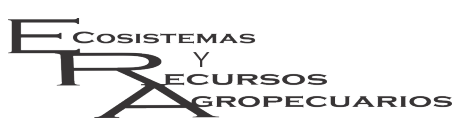

amplia distribución geográfica (Castro et al. 2011), característica debida a su capacidad de soportar condiciones adversas; lo que incrementa de forma rápida sus poblaciones (Cepeda 2016). Este fitonematodo, penetra la raíz desde el suelo, succionando el contenido celular, donde inyecta hormonas que desequilibran el desarrollo y la reproducción celular, causando hipertrofia e hiperplasia que dan origen a los síntomas típicos, como la presencia de agallas - nódulos que indican el síntoma visible de la infección primaria (Cepeda 2016). La infección del nematodo, se manifiesta en la planta como síntoma de deficiencia de agua, debido al daño en la raíz; la cual se pudre debido a la acción de microorganismos saprofitos; lo que se traduce en un menor desarrollo de la planta y aparición de síntomas como enanismo, clorosis, marchitez, falta de vigor, caída de flor y bajo rendimiento (Abad et al. 2009).

Para el manejo de nematodos, se han utilizado métodos físicos, culturales, químicos y una combinación de ellos. No obstante, el control químico es el método más utilizado, por su eficacia (Lamovšek et al. 2013). Particularmente el control del nematodo agallador con nematicidas químicos, ha sido mediante la aplicación al suelo, de fumigantes y no fumigantes de amplio espectro y fitotoxicidad (Giannakou y Karpouzas 2003). El empleo de nematicidas convencionales, ha sido restringido por los efectos nocivos al ambiente y a la salud humana (Huang et al. 2014). Además el costo, la residualidad y la fitotoxicidad de los nematicidas para el cultivo, limitan su aplicación; por lo que es se requiere buscar alternativas ecológicas para combatir a los nematodos fitopatógenos (Collange et al. 2011, Hernández et al. 2015).

Las plantas son capaces de protegerse de las plagas por sí mismas, debido a que sintetizan una gran variedad de metabolitos secundarios relacionados con mecanismos de defensa, con propiedades insecticidas, acaricidas y nematicidas (Sosa y Tonn 2008). Estas substancias comprenden más de 30 000 compuestos diferentes, como los triterpenos, saponinas, fitoesteroles y/o sus precursores, que al ser liberados influyen en la presencia de los nematodos alrededor de la planta (Montes-Belmont y
Cepeda-Siller et al. Toxicidad de extractors contra Meloidogyne incognita Ecosist. Recur. Agropec. 5(13):143-148,2018

Flores-Moctezuma 2011). Por lo que se reconocen diversas plantas con actividad nematicida (Quevedo et al. 2010), como el nogal pecanero (Carya illinoinensis (Wangenh) K. Koch), para el control de M. incognita (Garrido et al. 2014). En el nogal se encuentran sustancias fitoquímicas, en las hojas como las naftoquinonas, taninos, inositol, derivados flavonoides, ácidos fenol carboxílicos y ácido ascórbico; en el pericarpio de los frutos se encuentran ácidos orgánicos, taninos y naftoquinonas; en los cotiledones ácidos grasos insaturados; en el tegumento, polifenoles y taninos, y en la nuez, vitaminas $A, B, C$ y $E$, sales minerales y yodo (Caglarirmak 2003).

El control biorracional con extractos vegetales es una alternativa prometedora, que permite el desarrollo de una agricultura más rentable y no contaminante del ambiente (Molina 2001). Por lo anterior, el objetivo del estudio fue evaluar la actividad toxica de extractos de nogal pecanero (C. illinoinensis), sobre el nematodo agallador $M$. incognita en plantas de tomate, en condiciones de invernadero.

\section{MATERIALES Y MÉTODOS}

La investigación se realizó en el Laboratorio de Nematología y en el área de invernaderos del Departamento de Parasitología de la Universidad Autónoma Agraria Antonio Narro (UAAAN). Se ubica en Saltillo, Coahuila de Zaragoza, México a $25^{\circ} 22^{\prime \prime}$ LN y $101^{\circ} 02^{\prime \prime}$ LO, a una altitud de 1742 msnm.

Los extractos vegetales de cáscara acuosa y ruezno etanólico de nogal pecanero fueron proporcionados por la empresa Fitokimica Industrial de México. A partir de nódulos característicos de $M$. incognita obtenidos de tubérculos de papas de la variedad Alpha, cosechadas en la región de Navidad, Galeana, Nuevo León, México; se obtuvieron hembras y masas de huevos. Las masas de huevos se desinfectaron con hipoclorito de sodio al $1 \%$ por 1 min y se lavaron tres veces con agua destilada estéril, para luego pasar las masas de huevos a una cámara bioclimática a temperatura de $25^{\circ} \mathrm{C}$, hasta 


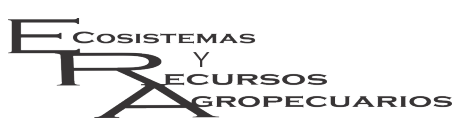

la eclosión del $95 \%$ de los huevos y emergencia de los juveniles de segundo estadio. De estos juveniles, se seleccionaron 2800 individuos, que se separaron en grupos de 100 en una placa de 28 cavidades. En las hembras adultas se realizaron cortes perineales, siguiendo la técnica de Taylor y Netscher (1974) para corroborar la especie, utilizando las claves taxonómicas de Eisenback et al. (1981).

Tabla 1. Extractos de Carya illinoinensis para evaluar la toxicidad contra Meloidogyne incognita en plántulas de tomate en invernadero.

\begin{tabular}{lcc} 
No. & Tratamientos & Concentración \\
\hline 1 & Ruezno etanólico & $1: 50$ \\
2 & Ruezno etanólico & $1: 75$ \\
3 & Cáscara acuosa & $1: 50$ \\
4 & Cáscara acuosa & $1: 75$ \\
5 & Ruezno acuoso & $1: 50$ \\
6 & Ruezno acuoso & $1: 75$ \\
7 & Agua destilada & Testigo \\
\hline Concentración 1:50=1.0 $\mathrm{mL}$ de extracto en \\
$50 \mathrm{~mL}$ de agua destilada, Concentración \\
$1: 75=1.0 \mathrm{~mL}$ de extracto en $75 \mathrm{~mL}$ de agua \\
destilada.
\end{tabular}

Se sembró semilla certificada de tomate variedad Pony Express $F 1$, en sustrato estéril de Peat moss-perlita, en proporción 3:1 e hidratado con agua destilada. A los $51 \mathrm{~d}$ después de la siembra, en plántulas con altura promedio de $10.6 \mathrm{~cm}$, se realizó el trasplante a vasos de poliestireno con 100 g Peat moss-perlita (3:1) como sustrato; para luego con una micropipeta inocular 100 juveniles de $M$. incognita (población inicial, $\mathrm{Pi}$ ). En el invernadero, los vasos de poliestireno se distribuyeron al azar en una cama alta de cultivo de $3 \times 1 \mathrm{~m}$ de largo y ancho $\times 0.80 \mathrm{~m}$ de altura, con distancia entre vasos de $10 \mathrm{~cm}$. A los $10 \mathrm{~d}$ después del trasplante, con apoyo de una micropipeta se aplicó $1 \mathrm{~mL}$ del tratamiento al sustrato (Tabla 1), estableciendose siete tratamientos con cuatro repeticiones, bajo un diseño en bloques completos al azar. La unidad experimental se formó por una planta de tomate. El experimento se realizó bajo condiciones controladas a temperatura de $24 \pm 2{ }^{\circ} \mathrm{C}$, humedad relativa de $60 \pm 10 \%$ y ventilación automatizada para reducir el calor y renovar el suministro de bióxido de carbono. Los riegos se realizaron cada tercer día con base en los requerimientos de la planta. También se realizó una aplicación de fertilizante foliar FertiPLUS+, a dosis de $2 \mathrm{~L} \mathrm{ha}^{-1}$, a los $15 \mathrm{~d}$ después del transplante con una bomba de mano de $2 \mathrm{~L}$.

La evaluación de la actividad nematicida de los extractos se llevó a cabo a los 30 d después de establecido el ensayo, que corresponde a un ciclo biológico normal de $M$. incognita, mediante la valoración de la densidad de población de juveniles de segunda generación, se realizó el conteo de la población final (Pf). Para lo cual se recuperó el sustrato de las plantas infectadas con nematodos y se colocó en embudo de Baerman, a las 48 h después de colocar el sustrato en el embudo, se procedió a realizar el conteo de la población final de nematodos juveniles de segundo estadio (vivos) y se obtuvo la reducción poblacional mediante la diferencia entre la población inicial al inicio del experimento (100 juveniles) y población final con la fórmula de Seinhorst (1970): I= Pf/Pi. Dónde: I, es el índice de incremento de la población, Pf es la población final y $\mathrm{Pi}$ es la población inicial.

La eficiencia de los tratamientos se determinó con la fórmula de Henderson-Tilton (1955): $\mathrm{E}=(1-\mathrm{Pfa} / \mathrm{Pia} \times \mathrm{Pib} / \mathrm{Pfb}) \times 100$. Dónde: $\mathrm{E}$ es la eficiencia de los tratamientos, Pia es la población inicial del tratamiento, $\mathrm{Pfa}$ es la población final del tratamiento, $\mathrm{Pib}$ es la población inicial del tratamiento testigo y $\mathrm{Pfb}$ es la población final del tratamiento testigo. La reducción de la población se analizó con análisis de varianza y la comparación de medias se realizó con la prueba de Tukey $(\mathrm{p}<$ $0.05)$, los análisis se realizaron con el software estadístico SAS 9.0.

\section{RESULTADOS Y DISCUSIÓN}

\section{Reducción de la población del nematodo agallador}

La aplicación de extractos de nogal pecanero tuvo efecto significativo $(p<0.05)$ en el control del nematodo agallador $M$. incognita, presentando mayor disminución de la población de nematodos el extracto de ruezno acuoso con 99 y $97 \%$ de control en las concentraciones $1: 50$ y $1: 75$, respectivamente; 
Tabla 2. Población inicial, población final, reducción de la población e índice de incremento poblacional del nematodo agallador Meloidogyne incognita y eficiencia de los tratamientos evaluados.

\begin{tabular}{cccccc}
\hline \multirow{2}{*}{ Tratamientos } & \multicolumn{4}{c}{ Densidad de población } & Eficiencia (\%) \\
\cline { 2 - 5 } & $\begin{array}{c}\text { Población } \\
\text { inicial (Pi) }\end{array}$ & $\begin{array}{c}\text { Población } \\
\text { final (Pf) }\end{array}$ & $\begin{array}{c}\text { Reducción de } \\
\text { la Población (\%) }\end{array}$ & $\begin{array}{c}\text { Indice de } \\
\text { incremento }\end{array}$ & \\
\hline Ruezno etanólico & 100 & 12.75 & $87.25^{a b}$ & 0.127 & 95.19 \\
Ruezno etanólico & 100 & 29.00 & $71.00^{a b}$ & 0.290 & 89.07 \\
Cáscara acuosa & 100 & 42.00 & $58.00^{b c}$ & 0.420 & 84.16 \\
Cáscara acuosa & 100 & 70.50 & $29.50^{c}$ & 0.705 & 73.42 \\
Ruezno acuoso & 100 & 1.00 & $99.00^{a}$ & 0.010 & 99.62 \\
Ruezno acuoso & 100 & 3.00 & $97.00^{a}$ & 0.030 & 98.87 \\
Agua destilada & 100 & 265.25 & $0.00^{d}$ & 2.652 & 00.00 \\
\hline Reducción de la poblacional con la misma letra, no son significativamente diferentes (Tukey $<0.05)$.
\end{tabular}

no permitiendo este extracto que la densidad de población se incrementará, de 0.01 a 0.03 veces la población inicial. En efectividad de control le siguió el extracto de ruezno etanólico, con un índice de incremento de la población de 0.127 y 0.290 veces la población inicial en las concentraciones $1: 50$ y $1: 75$, respectivamente (Tabla 2 ). Lo que indica que el tratamiento redujo la población de nematodos en 87.25 y $71.00 \%$, mientras que el extracto de cáscara acuosa presentó menor control de nematodos, con incremento de la población inicial de 0.420 veces en la concentración 1:50 y reducción de la población del $58 \%$, mientras que la concentración $1: 75$ tuvo un incremento de la población inicial de 0.705 veces, lo que indica una reducción de $29.5 \%$ de la población de $M$. incognita. Para el tratamiento testigo, sin control nematicida, se observó un incremento de la población de nematodos de 2.652 veces, permitiendo a la población aumentar 165.25 nematodos más que la población inicial $(\mathrm{g} l=6,27 ; \mathrm{F}=69.44$, $p<0.0001)$. Estos resultados son similares a los reportados por Dama (2002), quien tuvo un $100 \%$ de mortalidad de la población de Meloidogyne javanica in vitro con el compuesto orgánico naftalina, que se extrae del nogal. También concuerdan con lo encontrado por Garrido et al. (2014), quienes evaluaron bajo condiciones de laboratorio el control de $M$. incognita con extractos de nogal pecanero, reportando mayor actividad nematicida con el $89 \%$ de mortalidad el extracto de ruezno acuoso.

\section{Eficiencia de los tratamientos}

La eficiencia de los tratamientos (Tabla 2), muestra que los extractos de nogal pecanero tienen una alta eficiencia en el control de $M$. incognita, fluctuando entre el $73.42 \%$ en el extracto de cáscara acuosa en concentración $1: 75$, del $84.16 \%$ en el extracto con cáscara acuosa en concentración $1: 50,89.07 \%$ en el extracto de ruezno etanólico en concentración de 1:75. En tanto que en el ruezno etanólico en concentración 1:50 se tuvo una eficiencia del $95.19 \%$, los extractos de ruezno acuoso con la mayor eficiencia de control con el 99.62 y $98.87 \%$ se tuvo en las concentraciones 1:50 y 1:75 respectivamente. Los resultados indican que los extractos a base de nogal pecanero, presentan características nematicidas sobre $M$. incognita, con eficiencia variable en el control del nematodo, mostrando mayor efectividad el extracto acuoso de ruezno, lo que es consistente con lo reportado por Vinueza et al. (2006) quienes reportan efectividad de extractos acuosos de distintas plantas sobre juveniles de Meloidogyne spp.

El extracto de nogal pecanero tuvo una respuesta favorable en el control del nematodo agallador $M$. incognita en las concentraciones evaluadas, reduciendo la densidad de población en las raíces de tomate. Este control con extractos vegetales puede ser utilizado en cualquier sistema de producción agrícola, por sus características orgánicas e inocuidad con el ambiente. El extracto de ruezno acuoso y ruezno etanólico presentan alta efectividad de control sobre $M$. incognita, los cuales reúnen condiciones para ser utilizados en su control. 


\section{LITERATURA CITADA}

Abad PP, Castagnone-Sereno MN, Rosso JA, Engler B, Favery B (2009) Invasion, Feeding and Development. In: Moens M, Perry MN, Starr JL (Eds) Root-knot Nematodes. CAB International. pp: 163-164.

Caglarirmak N (2003) Biochemical and physical properties of some walnut genotypes (Juglans regia L.). Die Nahrung 47: 28-32.

Castro L, Flores L, Uribe L (2011) Efecto del vermicompost y quitina sobre el control de Meloidogyne incognita en tomate a nivel de invernadero. Agronomía Costarricense 35: 21-32.

Cepeda SM (2016) Nematología Agrícola. $2^{d a}$. reimpresión. Ed. Trillas, México. 304p.

Collange B, Navarrete M, Peyre G, Mateille T, Tchamitchian M (2011) Root-knot nematode (Meloidogyne) management in vegetable crop production: the challenge of an agronomic system analysis. Crop Protection 30: 1251-1262.

Dama LB (2002) Effect of naturally occurring napthoquinones on root-knot nematode Meloidogyne javanica Indian Phytopathology 55: 67-69.

Eisenback D, Hirschmann J, Sasser H, Triantaphyllou A (1981) Guide to the four most common species of root-knot nematodes (Meloidogyne sp.), with a pictorial key. The Department of Plant Pathology and Genetics, North Carolina State University, United States Agency for International Development. Raleigh, North Carolina, USA. 48p.

Garrido CF, Cepeda SM, Hernández CFD, Ochoa FYM, Cerna CE, Morales ADM (2014) Efectividad biológica de extractos de Carya illinoensis, para el control de Meloidogyne incognita. Revista Mexicana de Ciencias Agrícolas 5: 1317-1323.

Giannakou IO, Karpouzas DG (2003) Evaluation of chemical and integrated strategies as alternatives to methyl bromide for the control of root-knot nematodes in Greece. Pest Management Science 59: 883-892.

Henderson CF, Tilton EW (1955) Tests with acaricides against brown wheat mite. Journal of Economic Entomology 48: 157-161.

Hernández JA, Cepeda SM, Gallegos MG, Chacón HJC, Ordaz SS, González RA (2015) Actividad nematicida de productos orgánicos comerciales, contra Ditylenchus dipsaci (Tylenchida: Anguinidae) bajo condiciones de laboratorio. Revista Mexicana de Ciencias Agrícolas. Pub. Esp. 11: 2247-2252.

Huang WK, Sun JH, Cui JK, Wang GF, Kong LA, Peng H, et al. (2014) Efficacy evaluation of fungus Syncephalastrum racemosum and nematicide avermectin against the root-knot nematode Meloidogyne incognita on cucumber. PLoS ONE 9: e89717.

Jones JT, Haegeman A, Danchin EGJ, Gaur HS, Helder J, Jones MGK, et al. (2013) Top 10 plant-parasitic nematodes in molecular plant pathology. Molecular Plant Pathology 14: 946-961.

Lamovšek J, Urek G, Trdan S (2013) Biological Control of Root-Knot Nematodes (Meloidogyne spp.): Microbes against the Pests. Acta Agriculturae Slovenica 101: 263-275.

Molina N (2001) Uso de extractos botánicos en control de plagas y enfermedades. Avances en el fomento de productos fitosanitarios no-sintéticos. Manejo Integrado de Plagas 59: 76-77.

Montes-Belmont R, Flores-Moctezuma HE (2011) La alelopatía como base científica para el manejo de nematodos fitoparásitos. En: Rodríguez-Hernández C, López-Olguín JF, Aragón-García A (Eds) Alternativas ecológicas contra plagas. Agricultura sostenible. Colegio de Postgraduados, Benemérita Universidad Autónoma de Puebla. México. pp: 167-182. 
Nicol JM, Turner SJ, Coyne DL, den Nijs L, Hockland S, Tahna Maafi Z (2011) Current nematode threats to world agricultura. In: Jones J, Gheysen G, Fenoll C (Eds) Genomics and molecular genetics of plant-nematode interactions. Springer. Dordrecht, Holanda. pp. 21-43.

Quevedo O, Crozzoli R, Perichi G (2010) Uso de extractos acuosos y etanólicos de plantas para el control de Meloidogyne enterolobii (Nematoda: Tylenchida). Fitopatología Venezolana 23: 45-53.

SAGARPA-SIAP (2016) Producción agrícola de tomate. Secretaria de Agricultura, Ganadería, Desarrollo Rural, Pesca y Alimentación Servicio de Información Agroalimentaria y Pesquera. México. http://www.siap. gob.mx/cierre-de-la-produccion-agricola-por-estado/. Fecha de consulta: 20 de junio de 2016.

Seinhorst JW (1970) Dynamic of populations of plant parasitic nematodes. Annual Review of Phytopathology 8: $131-156$.

Sosa ME, Tonn CE (2008) Plant secondary metabolites from Argentinean semiarid lands: bioactivity against insects. Phytochemical Review 7: 3-24.

Taylor DP, Netscher C (1974) An improved technique for preparing perineal patterns of Meloidogyne spp. Nematológica 20: 268-269.

Vinueza PSM, Crozzoli R, Perichi G (2006) Evaluación in vitro de extractos acuosos de plantas para el control del nematodo agallador Meloidogyne incognita. Fitopatología venezolana 19: 26-31. 